\title{
BCG Vaccination in Early Childhood and Risk of Atopic Disease: A Systematic Review and Meta-Analysis
}

\author{
Keyu Zhao, ${ }^{1}$ Phoebe Miles, ${ }^{2}$ Xinyu Jiang, ${ }^{1}$ Qiongyan Zhou, ${ }^{1}$ Chao Cao, ${ }^{3}$ Wei Lin, \\ Richard Hubbard, ${ }^{4}$ Panfeng Fu ${ }^{\oplus},{ }^{5}$ and Suling $\mathrm{Xu}^{1}$ \\ ${ }^{1}$ Department of Dermatology, The Affiliated Hospital of Medical School, Ningbo University, 247 Renmin Road, \\ Ningbo 315000, China \\ ${ }^{2}$ Division of Humanities and Social Sciences, University of Nottingham, 199 Taikang East Road, Ningbo 315000, China \\ ${ }^{3}$ Department of Respiratory Medicine, Ningbo First Hospital, 59 Liuting Road, Ningbo 315000, China \\ ${ }^{4}$ Division of Epidemiology and Public Health, University of Nottingham, 199 Taikang East Road, Ningbo 315000, China \\ ${ }^{5}$ The Center of Medical Research, The Affiliated Hospital of Medical School, Ningbo University, 247 Renmin Road, \\ Ningbo 315000, China
}

Correspondence should be addressed to Panfeng Fu; panfengfu@hotmail.com

Received 16 June 2021; Revised 19 October 2021; Accepted 23 October 2021; Published 24 November 2021

Academic Editor: Isabella Annesi Maesano

Copyright (c) 2021 Keyu Zhao et al. This is an open access article distributed under the Creative Commons Attribution License, which permits unrestricted use, distribution, and reproduction in any medium, provided the original work is properly cited.

Background. Several large-scale studies suggest that Bacille Calmette-Guerin (BCG) vaccination in early childhood may reduce the risk of atopic diseases, but the findings remain controversial. Here, we aimed to investigate the potential correlation between early childhood BCG vaccination and the risk of developing atopic diseases. Methods. Eligible studies published on PubMed, EMBASE, and Cochrane CENTRAL were systematically sourced from 1950 to July 2021. Studies with over 100 participants and focusing on the association between BCG vaccine and atopic diseases including eczema, asthma, and rhinitis were included. Preliminary assessment of methods, interventions, outcomes, and study quality was performed by two independent investigators. Odds ratio (OR) with $95 \%$ confidence interval (CI) was calculated. Random effects of the meta-analysis were performed to define pooled estimates of the effects. Results. Twenty studies with a total of 222,928 participants were selected. The quantitative analysis revealed that administering BCG vaccine in early childhood reduced the risk of developing asthma significantly (OR $0.77,95 \%$ CI 0.63 to 0.93 ), indicating a protective efficacy of $23 \%$ against asthma development among vaccinated children. However, early administration of BCG vaccine did not significantly reduce the risk of developing eczema (OR 0.94, 95\% CI 0.76 to 1.16 ) and rhinitis (OR $0.99,95 \%$ CI 0.81 to 1.21). Further analysis revealed that the effect of BCG vaccination on asthma prevalence was significant especially in developed countries (OR 0.73 , 95\% CI 0.58 to 0.92 ). Conclusion. BCG vaccination in early childhood is associated with reduced risk of atopic disease, especially in developed countries.

\section{Introduction}

Allergy is malfunctioned immune response caused by various foreign antigens, which can increase the risk of asthma, atopic dermatitis (eczema), and rhinitis (hay fever) [1]. Atopic diseases are characterized by the incubation period between initial exposure (sensitization) and symptoms (elicitation) that will develop in subsequent exposures and may involve IgE and/or non-IgE-mediated responses. An IgE-mediated allergic reaction (sometimes called immediate-type hypersensitivity (type I)) involves the production of Th2 cytokines, which initiate IgE production by B cells [2]. Increased prevalence in allergic and autoimmune diseases has been appreciated worldwide, especially for the Western countries where the prevalence is as high as $20 \%$ [3], and one in three children in these countries [4] are affected by atopic diseases. Atopic diseases also have been positioned as the most common chronic disease, which greatly compromised life quality of patient and impose great social and economic impacts on both 
individuals and their families. Among patients with atopic disease, pediatric patients are affected greatly as they have high prevalence [5]. A recent register-based study showed that the lifetime prevalence of asthma and allergic rhinitis at age 10 was $15.6 \%$ and $20.4 \%$, respectively [6].

Vaccination is used worldwide for preventing infectious diseases [7]. Childhood vaccination plays a crucial role in the early development of the immune system [8]. Atopic disease usually develops in the early life of children, whose immune system is under development [9]. There is evidence revealing the relationship between vaccination and the risk of atopic disease [10], and many publications have investigated this hypothesis [11-15]. Among those relationships, the most investigated one is the relationship between BCG vaccine and atopic disease, but the results remain controversial. National BCG vaccination programs are standard for childhood immunization in most countries apart from Western European counties and North American countries to prevent tuberculosis [16]. Th2 immunologic response is inhibited by BCG. Antagonism of atopy by BCG has been observed in both human and animal models. Considering these characteristics, BCG is considered to be a therapeutic model for investigating the consequence of early-life stimulation of TH1 cells [17]. Recently, BCG is shown to have nonspecific beneficial effects on early immune system, reducing infant hospitalization [18]. However there were conflicting results between BCG and childhood atopic disease [19]. To clarify the relationship between BCG and childhood atopic disease, we conducted a meta-analysis of studies. We examined the association between BCG vaccine and atopic disease incidence and explored the implications of available literature data for clinical practice and future examination.

\section{Methods}

2.1. Strategy of Literature Search. Our systematic review was performed and reported in accordance with Meta-analysis Of Observational Studies in Epidemiology (MOOSE) [20] and the Preferred Reporting Items for Systematic Reviews and Meta-Analyses (PRISMA) [21] guidelines.

We performed a systematic literature search in PubMed/ Medline (1950 to Jul 2021), EMBASE (1980 to Jul 2021), and Cochrane CENTRAL (1950 to Jul 2021) for association between BCG vaccination and atopic disease by using relevant keywords including asthma, eczema, rhinitis, BCG vaccines, and other synonyms. The search strategy suitable for PubMed is provided in Appendix 1. The analysis was restricted to studies published in English. We screened bibliographies of relevant review articles to ensure that all relevant studies were included.

2.2. Study Selection. Each study was first selected based on their titles and abstracts (when available) by two independent investigators at the same time. Then, they retrieved full texts and performed further screening when studies were deemed eligible. Studies had to be cohort studies with information of authors, year, geographical area, study design, sample size, exposure (age of BCG vaccination, method of assessment), and outcome (confirmed diagnosis of specific atopic disease). Case reports, preclinical studies, and some studies without confirmed diagnosis of a specific atopic disease will not be used for this study. Disagreements were resolved by discussion and, if necessary, in consultation with a third senior investigator.

2.3. Quality Assessment. Quality of all included trials was assessed by two authors independently by using the STROBE checklist for cohort study and JADAD scale for RCT study. The risk of bias in each domain was judged as low, high, or unclear. The overall risk of bias in a study was classified as low if all domains had low risk; as high if one or more domains had high risk; or as unclear otherwise. Based on these standards, we defined the studies into the following three grades: A, high quality and low risk of bias (scored $\geq 66.6 \%$ ); $\mathrm{B}$, moderate quality and moderate risk of bias (scored 33.3-66.6\%); and C, low quality and high risk of bias $(<33.3 \%)$. Discrepancy in quality assessment between the reviewers was resolved by discussion with involvement of a third senior investigator if necessary.

2.4. Data Extraction. Two authors extracted data independently using a standard data extraction form. The following baseline characteristics were extracted from the included studies: first author, year of publication, study design, and location in which the study was performed, number of included participants, and diagnosis of atopic disease. Studies were excluded if any of the above information is not available.

2.5. Statistical Analysis. We used R (version 3.6.3) to perform the data analysis; odds ratios (ORs) and their associated $95 \%$ confidence intervals (CI) were used to assess the strength of association between BCG vaccination in early life and the risk of getting atopic disease. Statistical significance was defined at $p<0.05$ [22]. $I^{2}$ statistic were used for investigating heterogeneity, and statistical heterogeneity was defined at $I^{2}>50 \%$. We used random-effects modeling to perform the meta-analysis for all pooling. In case heterogeneity exists, analysis to investigate whether the heterogeneity is related to the participant's race will be performed. Different ethnic background in different continents may be considered potentially important to heterogeneity because of living habit diversity. Funnel plots were used to display the publication bias graphically, both specifically and officially with Egger's test.

\section{Results}

Our search strategy generated 4127 citations from 3 databases. Among them, 3946 articles were removed after exclusion of duplicates and screening of titles and abstracts. Of the remaining 181 studies, 161 studies were excluded after reviewing the full text. In total, 20 articles including 222928 participants met the inclusion criteria and were included in 
PRISMA 2020 flow diagram for new systematic reviews which included searches of databases and registers only

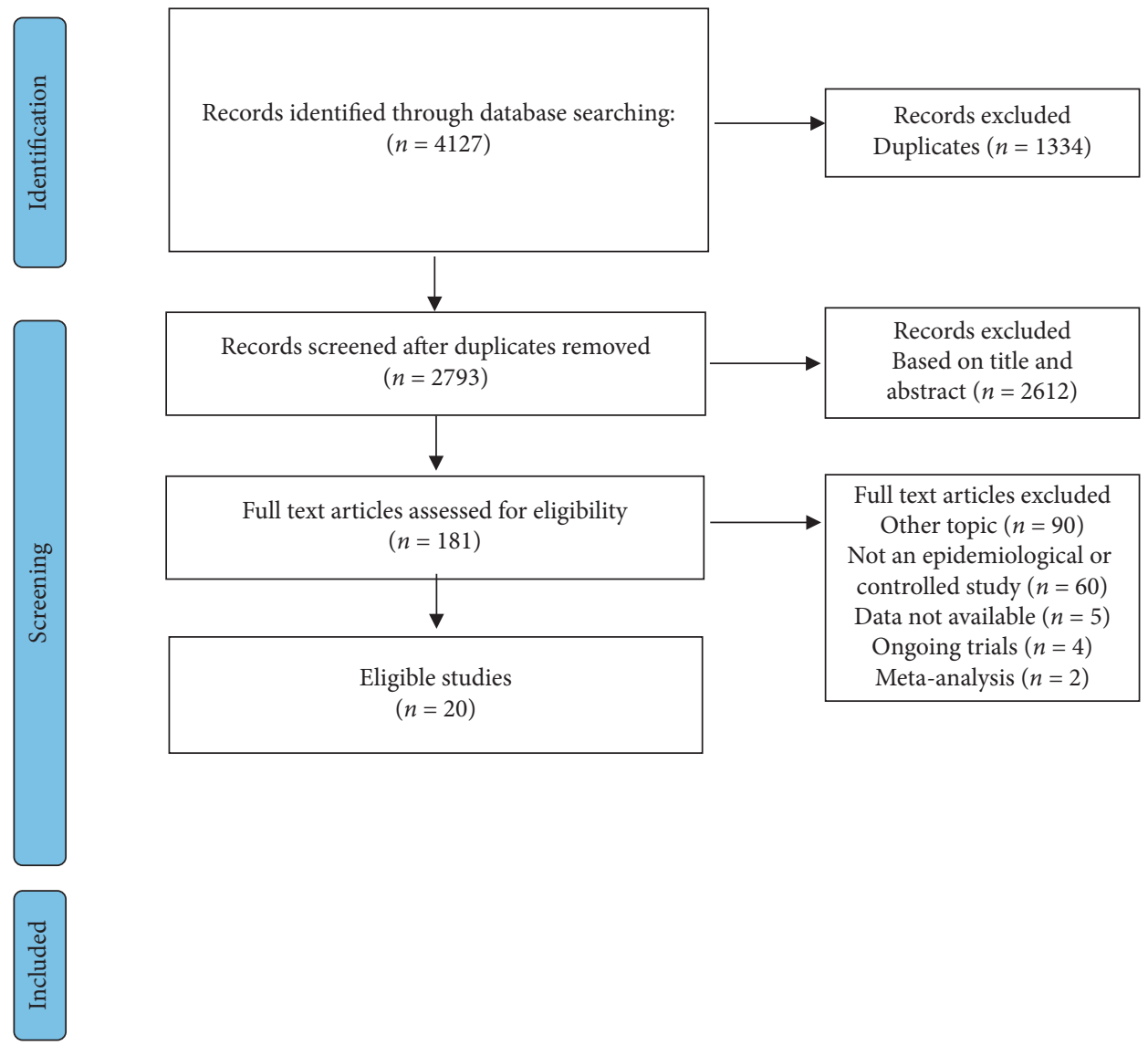

FIgURE 1: The Preferred Reporting Items for Systematic Review and Meta-Analysis (PRISMA) flow chart for the included studies.

the meta-analysis [11, 23-41].The flow diagram of trial identification and selection is shown in Figure 1. Descriptions and baseline characteristics of included studies are detailed in Table 1. No problems were encountered with participant data deficiency during the data integrity check.

Four studies were conducted in developing countries, and sixteen studies were in developed countries. None of the included studies were at low risk of bias (rated A) as all trials had an element of pragmatism in using different methods. Fifteen studies including $87 \%$ participants were deemed to be at moderate risk of bias (rated B). We judged five studies including only $13 \%$ participants to have high risk of bias (rated C) in the field of study participation or statistical reporting.

3.1. Association of BCG Vaccination in Childhood with Incidence of Atopic Disease. In the pooled analysis, we found that participants receiving BCG in childhood associated with a lower risk of atopic disease than that of the non-BCG group $(\mathrm{OR}=0.87,95 \% \mathrm{CI} 0.77$ to 0.99 ; Figure 2$)$. Nineteen studies involving 80922 participants reported the relationship between BCG vaccination and the risk of asthma. Compared with nonBCG group, individuals received BCG in early childhood were associated with a significantly reduced risk of asthma $(\mathrm{OR}=0.77,95 \%$ CI 0.63 to 0.93 ; Figure 2$)$. Thirteen studies including 72646 participants reported the association between eczema and BCG vaccine, and twelve studies including 69360 participants studied rhinitis and BCG vaccine. Compared with the control group, BCG vaccine showed no significant effect on preventing eczema and rhinitis $(\mathrm{OR}=0.94$ and $0.99,95 \% \mathrm{CI}$ 0.76 to 1.16 and 0.81 to 1.21 , respectively; Figure 2).

3.2. Association between BCG Vaccination and Demographic Factors on the Risk of Atopic Disease. We evaluated the association between BCG vaccination and prevalence of asthma in terms of participants' demographics. Participants from developed countries were associated with a significantly lower risk of developing asthma when administered BCG vaccine in early childhood (pooled OR $=0.73,95 \%$ CI 0.58 to 0.92 ; Figure 3 ). In contrast, in developing countries, participants who received BCG vaccine in early childhood were not associated with a significantly reduced risk of allergic disease (pooled $\mathrm{OR}=0.86$, 95\% CI 0.69 to 1.07; Figure 3).

BCG vaccine was not associated with the risk of eczema in the subgroup analysis of different continents (Figure 4). Similar results were obtained in participants with rhinitis (Figure 5). These results of our study did not support an association of BCG vaccination with reduction in the risk of eczema or rhinitis in both developed and developing countries. 
TABLE 1: Characteristics of included studies.

\begin{tabular}{|c|c|c|c|c|c|c|}
\hline $\begin{array}{l}\text { Reference } \\
\text { (year of publication) }\end{array}$ & Design & Population studied & Country & $\begin{array}{l}\text { Sample size } \\
\text { (case/control) }\end{array}$ & Outcomes and method & $\begin{array}{l}\text { Quality } \\
\text { assessment }\end{array}$ \\
\hline Zein 2017 & $\begin{array}{l}\text { Retrospective } \\
\text { study }\end{array}$ & $\begin{array}{l}\text { Vaccination: } 32,900 \\
(42.9 \%) \text { individuals } \\
\text { received the vaccine } \\
\text { within their first year of } \\
\text { life, } 2,712(3.5 \%) \text { were } \\
\text { vaccinated later. Follow } \\
\text { up: children born at } 32 \\
\text { weeks of gestation until } \\
\text { 20n years Quebec, } \\
\text { Canada, } 1974 .\end{array}$ & $\begin{array}{l}\text { Developed } \\
\text { country }\end{array}$ & $893 / 750$ & $\begin{array}{l}\text { Asthma at least } 2 \text { asthma- } \\
\text { related medical services or } \\
\text { at least } 1 \text { asthma- related } \\
\text { hospitalization } \\
\text { (international } \\
\text { classification of diseases, } \\
\text { ninth revision, code } 493 \text { ) }\end{array}$ & B \\
\hline Alm 1997 & $\begin{array}{l}\text { Retrospective } \\
\text { study }\end{array}$ & $\begin{array}{l}\text { Vaccination: Before the } \\
\text { age of } 6 \text { months, follow } \\
\text { up: children at } 3-7 \text { years } \\
\text { Stockholm, Sweden } 1991\end{array}$ & $\begin{array}{l}\text { Developed } \\
\text { country }\end{array}$ & $216 / 358$ & $\begin{array}{l}\text { Eczema, asthma, rhinitis } \\
\text { atopic disease in history } \\
\text { and/or at the clinical } \\
\text { examination, as well as } \\
\text { skin prick test and IgE in a } \\
\text { blood sample }\end{array}$ & B \\
\hline Garcia 2005 & $\begin{array}{l}\text { Retrospective } \\
\text { study }\end{array}$ & $\begin{array}{l}\text { Vaccination: When the } \\
\text { children were born follow } \\
\text { up: children aged } 6 \text { and } 7 \\
\text { years old health centers in } \\
\text { Spain }\end{array}$ & $\begin{array}{l}\text { Developed } \\
\text { country }\end{array}$ & $6762 / 2828$ & $\begin{array}{l}\text { Eczema, asthma, rhinitis } \\
\text { the Spanish version of the } \\
\text { ISAAC phase III } \\
\text { questionnaire }\end{array}$ & B \\
\hline Gruber 2002 & $\begin{array}{l}\text { Retrospective } \\
\text { study }\end{array}$ & $\begin{array}{l}\text { Vaccination: When the } \\
\text { children were born follow } \\
\text { up: children who were } \\
\text { starting school (mean age } \\
6 \text { years) Germany, } 1994\end{array}$ & $\begin{array}{l}\text { Developed } \\
\text { country }\end{array}$ & $20383 / 18425$ & $\begin{array}{l}\text { Eczema, asthma, rhinitis a } \\
\text { mandatory health survey }\end{array}$ & B \\
\hline Marks 2003 & $\begin{array}{l}\text { Retrospective } \\
\text { study }\end{array}$ & $\begin{array}{l}\text { Vaccination: In the first } 8 \\
\text { weeks of life follow up: } \\
\text { yellow race children aged } \\
7 \text { to } 14 \text { years old Sydney, } \\
\text { Australia }\end{array}$ & $\begin{array}{l}\text { Developed } \\
\text { country }\end{array}$ & $309 / 442$ & $\begin{array}{l}\text { Eczema, asthma, rhinitis } \\
\text { subjects underwent } \\
\text { allergen SPTs, spirometry, } \\
\text { a methacholine inhalation } \\
\text { challenge test, a venous } \\
\text { blood collection for IgE } \\
\text { assay and lymphocyte } \\
\text { studies, and a TST }\end{array}$ & B \\
\hline Miyake 2008 & $\begin{array}{l}\text { Retrospective } \\
\text { study }\end{array}$ & $\begin{array}{l}\text { Vaccination: In infancy } \\
\text { according to school } \\
\text { records follow up: } 8-11 \\
\text { years of age Japan }\end{array}$ & $\begin{array}{l}\text { Developed } \\
\text { country }\end{array}$ & $5567 / 150$ & $\begin{array}{l}\text { Eczema, asthma, rhinitis a } \\
\text { self-administered } \\
\text { questionnaires included } \\
\text { questions on symptoms of } \\
\text { wheeze, asthma, atopic } \\
\text { eczema }\end{array}$ & B \\
\hline Linehan 2007 & $\begin{array}{l}\text { Retrospective } \\
\text { study }\end{array}$ & $\begin{array}{l}\text { Vaccination: Before the } \\
\text { age of } 12 \text { weeks followed } \\
\text { up: } 6-11 \text { years of age } \\
\text { health care center, } \\
\text { England }\end{array}$ & $\begin{array}{l}\text { Developed } \\
\text { country }\end{array}$ & $1332 / 1076$ & $\begin{array}{l}\text { Wheeze a parent- } \\
\text { completed questionnaire, } \\
\text { based on the international } \\
\text { study of asthma and } \\
\text { allergies in childhood } \\
\text { asthma questionnaire } 12 \\
\text { with some additional } \\
\text { questions }\end{array}$ & B \\
\hline da cunha 2004 & $\begin{array}{l}\text { Cross- } \\
\text { sectional }\end{array}$ & $\begin{array}{l}\text { Vaccination: When the } \\
\text { children were born } \\
\text { followed up: children of } \\
\text { 12-14 years Salvador, } \\
\text { Brazil }\end{array}$ & $\begin{array}{l}\text { Developing } \\
\text { country }\end{array}$ & $1089 / 523$ & $\begin{array}{l}\text { Eczema, asthma, rhinitis } \\
\text { based on self-reporting of } \\
\text { current allergy and } \\
\text { whether this was } \\
\text { accompanied by sneezing } \\
\text { and/or skin } \\
\text { manifestations }\end{array}$ & $\mathrm{C}$ \\
\hline
\end{tabular}


TABle 1: Continued.

\begin{tabular}{|c|c|c|c|c|c|c|}
\hline $\begin{array}{l}\text { Reference } \\
\text { (year of publication) }\end{array}$ & Design & Population studied & Country & $\begin{array}{l}\text { Sample size } \\
\text { (case/control) }\end{array}$ & Outcomes and method & $\begin{array}{c}\text { Quality } \\
\text { assessment }\end{array}$ \\
\hline Mommers 2004 & $\begin{array}{l}\text { Nested case- } \\
\text { control study }\end{array}$ & $\begin{array}{l}\text { Vaccination: When the } \\
\text { children were born } \\
\text { followed up: children at } \\
7-8 \text { years of age } \\
\text { Netherlands and } \\
\text { Germany }\end{array}$ & $\begin{array}{l}\text { Developed } \\
\text { country }\end{array}$ & Case:75 & $\begin{array}{l}\text { Asthma ISAAC } \\
\text { questionnaire and } \\
\text { questions on indoor } \\
\text { environment, specific IgE } \\
\text { was measured in the blood }\end{array}$ & $\mathrm{C}$ \\
\hline $\begin{array}{l}\text { Mohrenschlager } \\
2007\end{array}$ & $\begin{array}{l}\text { Cross- } \\
\text { sectional study }\end{array}$ & $\begin{array}{l}\text { Vaccination: Unknow } \\
\text { followed up: at 5-7 years } \\
\text { (preschool) augsburg } \\
\text { Germany }\end{array}$ & $\begin{array}{l}\text { Developed } \\
\text { country }\end{array}$ & $1219 / 247$ & $\begin{array}{l}\text { Eczema, asthma, rhinitis } \\
\text { skin prick test reaction } \\
\text { more than } 2 \mathrm{~mm}\end{array}$ & $\mathrm{C}$ \\
\hline Pahari 2002 & $\begin{array}{l}\text { Cross- } \\
\text { sectional study }\end{array}$ & $\begin{array}{l}\text { Vaccination: Unknow } \\
\text { follow up: children aged } \\
11-18 \text { years from } \\
\text { secondary school } \\
\text { England }\end{array}$ & $\begin{array}{l}\text { Developed } \\
\text { country }\end{array}$ & Case:308 & Asthma clinical symptom & $\mathrm{C}$ \\
\hline Thestesen 2018 & RCT & $\begin{array}{l}\text { Vaccination: When the } \\
\text { children were born follow } \\
\text { up: from birth to } 13 \\
\text { months of age Denmark }\end{array}$ & $\begin{array}{l}\text { Developed } \\
\text { country }\end{array}$ & $2052 / 1952$ & $\begin{array}{c}\text { Eczema the severity of } \mathrm{AD} \\
\text { was graded using } \\
\text { SCORAD }\end{array}$ & $\mathrm{B}$ \\
\hline Gruber 2001 & $\begin{array}{l}\text { Prospective } \\
\text { study }\end{array}$ & $\begin{array}{l}\text { Vaccination: Within their } \\
\text { first weeks of life follow } \\
\text { up: from birth to } 7 \text { years } \\
\text { old Freiburg, Germany }\end{array}$ & $\begin{array}{l}\text { Developed } \\
\text { country }\end{array}$ & $92 / 682$ & $\begin{array}{l}\text { Eczema, asthma, rhinitis } \\
\text { parents filled in a } \\
\text { questionnaire and gave a } \\
\text { structured interview about } \\
\text { their children } s \text { diseases } \\
\text { and atopic symptoms }\end{array}$ & $\mathrm{B}$ \\
\hline Soysal 2008 & $\begin{array}{l}\text { Retrospective } \\
\text { study }\end{array}$ & $\begin{array}{l}\text { Vaccination: Unknow } \\
\text { follow up: children with } \\
\text { recent household contact } \\
\text { to adult given a diagnosis } \\
\text { of sputum smear- } \\
\text { positive pulmonary TB at } \\
\text { one of } 7 \text { government- } \\
\text { funded TB clinics } \\
\text { istanbul }\end{array}$ & $\begin{array}{l}\text { Developing } \\
\text { country }\end{array}$ & $290 / 71$ & $\begin{array}{l}\text { Eczema, asthma, rhinitis } \\
\text { all children underwent } \\
\text { TST and chest } \\
\text { radiography, and gave 10- } \\
\text { ml of venous blood sample } \\
\text { for the RD1 ELISpot assay } \\
\text { and total serum IgE level }\end{array}$ & $\mathrm{B}$ \\
\hline Steenhuis 2008 & RCT & $\begin{array}{l}\text { Vaccination: When the } \\
\text { children were born } \\
\text { Follow up: high-risk } \\
\text { newborns in the pediatric } \\
\text { Utrecht, Netherlands }\end{array}$ & $\begin{array}{l}\text { Developed } \\
\text { country }\end{array}$ & $62 / 59$ & $\begin{array}{l}\text { Eczema, asthma rhinitis } \\
\text { an adapted version of the } \\
\text { british medical research } \\
\text { council questionnaire and } \\
\text { the Dutch version of the } \\
\text { european community } \\
\text { respiratory health survey } \\
\text { were used }\end{array}$ & $\mathrm{C}$ \\
\hline Wickens 2001 & $\begin{array}{l}\text { Case-control } \\
\text { study }\end{array}$ & $\begin{array}{l}\text { Vaccination: When the } \\
\text { children were born follow } \\
\text { up: children aged 6-7 and } \\
13-14 \text { from a random } \\
\text { selection of schools } \\
\text { Wellington, New Zealand }\end{array}$ & $\begin{array}{l}\text { Developed } \\
\text { country }\end{array}$ & $220 / 231$ & $\begin{array}{l}\text { Asthma the case-control } \\
\text { study was based on the } \\
\text { Wellington, New Zealand, } \\
\text { arm of the ISAAC }\end{array}$ & $\mathrm{C}$ \\
\hline Peleteiro 2018 & RCT & $\begin{array}{c}\text { Vaccination: All } \\
\text { individuals had been } \\
\text { vaccinated with BCG in } \\
\text { early childhoodFollow } \\
\text { up: healthy } \\
\text { undergraduate } \\
\text { studentsSalvador, Bahia, } \\
\text { Brazil }\end{array}$ & $\begin{array}{l}\text { Developing } \\
\text { country }\end{array}$ & $46 / 29$ & $\begin{array}{c}\text { Eczema, asthma rhinitis } \\
\text { sociodemographic and } \\
\text { clinical characteristics } \\
\text { were obtained using a } \\
\text { standard questionnaire } \\
\text { and the ISAAC } \\
\text { questionnaire. Total } \\
\text { serum IgE levels were } \\
\text { measured }\end{array}$ & $\mathrm{B}$ \\
\hline
\end{tabular}


TABLE 1: Continued.

\begin{tabular}{|c|c|c|c|c|c|c|}
\hline $\begin{array}{l}\text { Reference } \\
\text { (year of publication) }\end{array}$ & Design & Population studied & Country & $\begin{array}{l}\text { Sample size } \\
\text { (case/control) }\end{array}$ & Outcomes and method & $\begin{array}{c}\text { Quality } \\
\text { assessment }\end{array}$ \\
\hline Park 2015 & $\begin{array}{l}\text { Retrospective } \\
\text { study }\end{array}$ & $\begin{array}{c}\text { Vaccination: } \\
\text { unknowFollow up: adults } \\
\text { who underwent skin } \\
\text { pricktesting, Korea }\end{array}$ & $\begin{array}{l}\text { Developed } \\
\text { country }\end{array}$ & $135 / 40$ & $\begin{array}{l}\text { Asthma the results from } \\
\text { spirometry and bronchial } \\
\text { provocation testing } \\
\text { conducted within one } \\
\text { month from the date of } \\
\text { SPT }\end{array}$ & $\mathrm{C}$ \\
\hline Singh 2013 & $\begin{array}{l}\text { Cross- } \\
\text { sectional study }\end{array}$ & $\begin{array}{l}\text { Vaccination: } \\
\text { unknowFollow up: aged } \\
7-14 \text { years Chandigarh, } \\
\text { North India. }\end{array}$ & $\begin{array}{l}\text { Developing } \\
\text { country }\end{array}$ & $7792 / 1700$ & $\begin{array}{l}\text { Eczema, asthma rhinitis } \\
\text { symptoms were assessed } \\
\text { according to the ISAAC } \\
\text { phase II questionnaire } \\
\text { that asked for infor- } \\
\text { mation on demography, } \\
\text { symptoms of wheeze, } \\
\text { asthma, rhinitis, and } \\
\text { eczema, together with } \\
\text { various possible risk } \\
\text { factors }\end{array}$ & B \\
\hline Thestesen 2017 & $\mathrm{RCT}$ & $\begin{array}{l}\text { Vaccination: When the } \\
\text { children were born follow } \\
\text { up: newborns ( } 3-13 \\
\text { months) Denmark }\end{array}$ & $\begin{array}{l}\text { Developed } \\
\text { country }\end{array}$ & $2100 / 2071$ & $\begin{array}{l}\text { Asthma at } 3 \text { and } 13 \\
\text { months, the children were } \\
\text { invited for a clinical } \\
\text { examination at the study } \\
\text { site, where study staff } \\
\text { evaluated the child's } \\
\text { breathing and made an } \\
\text { auscultation }\end{array}$ & B \\
\hline
\end{tabular}

3.3. Publication Bias. The total publication bias was outlined in the funnel plot (Figure 6). Based on the funnel plots and Egger's test [42], it suggested that publication bias did not impact our estimates $(p=0.074)$.

\section{Discussion}

In this systematic review and meta-analysis, we revealed that BCG vaccine might be effective in preventing atopic disease, especially for asthma among developed countries. However, compared with asthma, the risk of developing atopic disease was not reduced for both eczema and rhinitis. In developing countries, there was no association between BCG vaccine and atopic diseases. As far as we know, this systematic review and meta-analysis is the latest study investigating the association between BCG vaccination and atopic diseases.

In this study, we found that receiving a BCG vaccine in early childhood especially reduced the risk of asthma. According to other studies on BCG vaccination and asthma, BCG vaccine, which was proved to prevent the inflammation caused by mycobacteria, has been tested to inhibit allergeninduced airway inflammation in a murine model of atopic asthma [43]. The immune balance was adjusted by BCG vaccine during Th1-like activity and decreasing the IL-4 and IL10 production. It was not clear how IFN- $\gamma$ influences Th2 responses, but it may involve macrophage activation and inhibition of Th2 lymphocytes development or change antigen presentation directly [44]. The mechanism of BCG vaccine on asthma was not as simple as changes in the Th1/Th2 balance [45], and we speculated that early childhood BCG vaccination accelerates the conversion from a Th2 to a Th1 type, therefore restraining the expression of atopy.

In developed countries, the association between BCG vaccination and reduction of the risk of asthma is more significant than the other developing countries of the world. This could be attributable to the fact that, in the regions other than developed countries, helminth infections and tuberculosis are endemic; therefore, the effects of BCG vaccine on reducing prevalence of allergic diseases were compromised [46]. The protection level of BCG vaccination is correlated to the gradient of exposure to environmental mycobacteria, a gradient from lower protection in countries close to the equator towards higher protection with increasing distance from the equator [47]. It is also reported that early-life events or diseases like perinatal circumstances or early allergen exposure would increase the prevalence of atopic diseases [48]. However, due to the limited number of studies included, heterogeneity might not be shown in the subgroup analysis of subjects at high risk or of developing countries. Based on this meta-analysis, the positive protective role of BCG vaccine in atopic disease requires further investigation, especially more cohort studies on children from high-risk areas.

As for rhinitis and eczema, there is no significant association, even though they may share similar genetic characteristics with asthma [49]. This is likely because some methodologic limitations may limit our interpretation of the findings. We expected that analyzing heterogeneity through technical means to analyze the original data might be helpful to rule out the reasons of the heterogeneity generated by specific studies. Due to the lack of information on the 


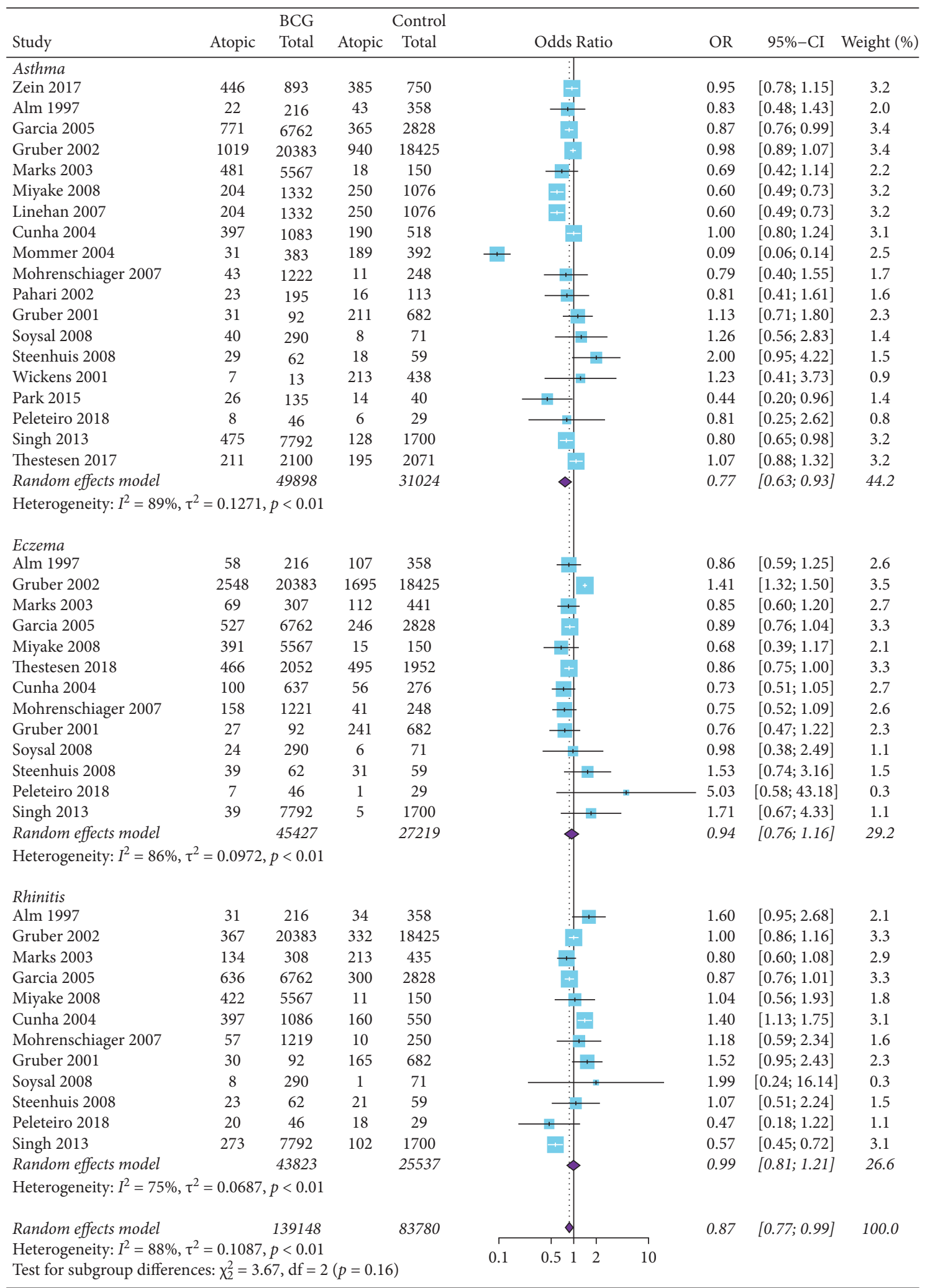

FIGURE 2: Forest plot of BCG and total atopic diseases. Random effects model forest plot shows ORs and 95\% CIs for the association between $\mathrm{BCG}$ vaccination and total atopic disease. $\mathrm{OR}=0.87,95 \% \mathrm{CI} 0.77$ to 0.99 . $\mathrm{M}-\mathrm{H}=$ Mantel-Haenszel; $\mathrm{OR}=\mathrm{odds}$ ratio; $\mathrm{CI}=$ confidence interval. 


\begin{tabular}{|c|c|c|c|c|c|c|c|c|c|}
\hline \multirow{3}{*}{$\begin{array}{l}\text { Study } \\
\text { developed country }\end{array}$} & \multirow{3}{*}{ asthma } & \multirow{3}{*}{$\begin{array}{l}\text { BCG } \\
\text { Total }\end{array}$} & \multirow{3}{*}{ asthma } & \multirow{2}{*}{\multicolumn{2}{|c|}{$\begin{array}{c}\text { Control } \\
\text { Total }\end{array}$}} & \multirow[b]{2}{*}{ Odds Ratio } & \multirow{3}{*}{ OR } & \multirow{3}{*}{$95 \%-\mathrm{CI}$} & \multirow[b]{2}{*}{ Weight (\%) } \\
\hline & & & & & & & & & \\
\hline & & & & & & & & & \\
\hline Zein 2017 & 446 & 893 & 385 & 750 & & 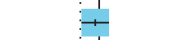 & 0.95 & {$[0.78 ; 1.15]$} & 7.1 \\
\hline Alm 1997 & 22 & 216 & 43 & 358 & & $\therefore$ & 0.83 & {$[0.48 ; 1.43]$} & 4.8 \\
\hline Garcia 2005 & 771 & 6762 & 365 & 2828 & & + & 0.87 & {$[0.76 ; 0.99]$} & 7.4 \\
\hline Gruber 2002 & 1019 & 20383 & 940 & 18425 & & $\vdots+$ & 0.98 & {$[0.89 ; 1.07]$} & 7.5 \\
\hline Marks 2003 & 481 & 5567 & 18 & 150 & & $\because$ & 0.69 & {$[0.42 ; 1.14]$} & 5.0 \\
\hline Miyake 2008 & 204 & 1332 & 250 & 1076 & & + & 0.60 & {$[0.49 ; 0.73]$} & 7.0 \\
\hline Linehan 2007 & 204 & 1332 & 250 & 1076 & & $\mp$ & 0.60 & {$[0.49 ; 0.73]$} & 7.0 \\
\hline Mommer 2004 & 31 & 383 & 189 & 392 & $\longrightarrow$ & & 0.09 & {$[0.06 ; 0.14]$} & 5.6 \\
\hline Mohrenschiager 2007 & 43 & 1222 & 11 & 248 & & \begin{tabular}{l|l}
$\div$ \\
\end{tabular} & 0.79 & {$[0.40 ; 1.55]$} & 3.9 \\
\hline Pahari 2002 & 23 & 195 & 16 & 113 & & $\div$ & 0.81 & {$[0.41 ; 1.61]$} & 3.9 \\
\hline Gruber 2001 & 31 & 92 & 211 & 682 & & 1 & 1.13 & {$[0.71 ; 1.80]$} & 5.3 \\
\hline Steenhuis 2008 & 29 & 62 & 18 & 59 & & + & 2.00 & {$[0.95 ; 4.22]$} & 3.6 \\
\hline Wickens 2001 & 7 & 13 & 213 & 438 & & $\begin{array}{c}\vdots \\
\vdots\end{array}$ & 1.23 & {$[0.41 ; 3.73]$} & 2.2 \\
\hline Park 2015 & 26 & 135 & 14 & 40 & & $+\quad \vdots$ & 0.44 & {$[0.20 ; 0.96]$} & 3.4 \\
\hline Thestesen 2017 & 211 & 2100 & 195 & 2071 & & $\vdots$ & 1.07 & {$[0.88 ; 1.32]$} & 7.0 \\
\hline Random effects model & & 40687 & & 28706 & & $<$ & 0.73 & {$[0.58 ; 0.92]$} & 80.7 \\
\hline \multicolumn{10}{|c|}{ Heterogeneity: $I^{2}=91 \%, \tau^{2}=0.1571, p<0.01$} \\
\hline \multicolumn{10}{|l|}{ developing country } \\
\hline Cunha 2004 & 397 & 1083 & 190 & 518 & & 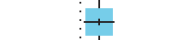 & 1.00 & {$[0.80 ; 1.24]$} & 7.0 \\
\hline Soysal 2008 & 40 & 290 & 8 & 71 & & $:]_{1}$ & 1.26 & {$[0.56 ; 2.83]$} & 3.3 \\
\hline Peleteiro 2018 & 8 & 46 & 6 & 29 & & 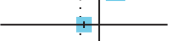 & 0.81 & {$[0.25 ; 2.62]$} & 2.0 \\
\hline Singh 2013 & 475 & 7792 & 128 & 1700 & & $\div$ & 0.80 & {$[0.65 ; 0.98]$} & 7.0 \\
\hline Random effects model & & 9211 & & 2318 & & a & 0.89 & {$[0.77 ; 1.03]$} & 19.3 \\
\hline \multicolumn{10}{|c|}{ Heterogeneity: $I^{2}=0 \%, \tau^{2}=0, p=0.40$} \\
\hline Random effects model & & 49898 & & 31024 & & $\dot{\theta}$ & 0.77 & {$[0.63 ; 0.93]$} & 100.0 \\
\hline $\begin{array}{l}\text { Heterogeneity: } I^{2}=89 \\
\text { Test for subgroup diffe }\end{array}$ & $\begin{array}{l}=0.1271 \\
\text { s: } \chi_{1}^{2}=2 .\end{array}$ & $\begin{array}{l}p<0.01 \\
05, \mathrm{df}=1\end{array}$ & $p=0.15$ & & 0.1 & 0.51 & & & \\
\hline
\end{tabular}

Figure 3: Forest plot of the association between BCG vaccination and asthma. Random effects model forest plot shows ORs and 95\% CIs for the association between BCG vaccination and asthma. $\mathrm{OR}=0.73,95 \%$ CI 0.58 to 0.92 . M-H= Mantel-Haenszel; OR=odds ratio; $\mathrm{CI}=$ confidence interval.

\begin{tabular}{|c|c|c|c|c|c|c|c|c|c|c|}
\hline Study & rhinitis & $\begin{array}{l}\text { BCG } \\
\text { Total }\end{array}$ & rhinitis & $\begin{array}{c}\text { Control } \\
\text { Total }\end{array}$ & & Odds Ratio & & OR & $95 \%-\mathrm{CI}$ & Weight (\%) \\
\hline \multicolumn{11}{|l|}{ developed country } \\
\hline Alm 1997 & 31 & 216 & 34 & 358 & & + & & 1.60 & {$[0.95 ; 2.68]$} & 7.4 \\
\hline Gruber 2002 & 367 & 20383 & 332 & 18425 & & & & 1.00 & {$[0.86 ; 1.16]$} & 13.8 \\
\hline Marks 2003 & 134 & 308 & 213 & 435 & & 4 & & 0.80 & {$[0.60 ; 1.08]$} & 11.3 \\
\hline Garcia 2005 & 636 & 6762 & 300 & 2828 & & + & & 0.87 & {$[0.76 ; 1.01]$} & 13.9 \\
\hline Miyake 2008 & 422 & 5567 & 11 & 150 & & $\frac{1}{1}$ & & 1.04 & {$[0.56 ; 1.93]$} & 6.1 \\
\hline Mohrenschiager 2007 & 57 & 1219 & 10 & 250 & & + & & 1.18 & {$[0.59 ; 2.34]$} & 5.4 \\
\hline Gruber 2001 & 30 & 92 & 165 & 682 & & 5 & & 1.52 & {$[0.95 ; 2.43]$} & 8.1 \\
\hline Steenhuis 2008 & 23 & 62 & 21 & 59 & & $\frac{1}{1}$ & & 1.07 & {$[0.51 ; 2.24]$} & 4.9 \\
\hline Random effects model & & 34609 & & 23187 & & & & 1.00 & {$[0.87 ; 1.16]$} & 70.8 \\
\hline \multicolumn{11}{|c|}{ Heterogeneity: $I^{2}=37 \%, \tau^{2}=0.0131, p=0.14$} \\
\hline \multicolumn{11}{|l|}{ developing country } \\
\hline Cunha 2004 & 397 & 1086 & 160 & 550 & & + & & 1.40 & {$[1.13 ; 1.75]$} & 12.6 \\
\hline Soysal 2008 & 8 & 290 & 1 & 71 & & & & 1.99 & {$[0.24 ; 16.14]$} & 0.8 \\
\hline Peleteiro 2018 & 20 & 46 & 18 & 29 & & + & & 0.47 & {$[0.18 ; 1.22]$} & 3.4 \\
\hline Singh 2013 & 273 & 7792 & 102 & 1700 & & + & & 0.57 & {$[0.45 ; 0.72]$} & 12.4 \\
\hline Random effects model & & 9214 & & 2350 & & & & 0.83 & {$[0.41 ; 1.70]$} & 29.2 \\
\hline \multicolumn{11}{|c|}{ Heterogeneity: $I^{2}=91 \%, \tau^{2}=0.3657, p<0.01$} \\
\hline Random effects model & & 43823 & & 25537 & & 1 & & 0.99 & {$[0.81 ; 1.21]$} & 100.0 \\
\hline \multicolumn{5}{|c|}{ Heterogeneity: $I^{2}=75 \%, \tau^{2}=0.0687, p<0.01$} & 0.1 & 0.5 & 10 & & & \\
\hline
\end{tabular}

FIGURE 4: Forest plot of the association between BCG vaccination and rhinitis. Random effects model forest plot shows ORs and 95\% CIs of the association between $\mathrm{BCG}$ vaccination and rhinitis. $\mathrm{OR}=0.99,95 \% \mathrm{CI} 0.81$ to 1.21 . $\mathrm{M}-\mathrm{H}=$ Mantel-Haenszel; OR=odds ratio; $\mathrm{CI}=$ confidence interval. 


\begin{tabular}{|c|c|c|c|c|c|c|c|c|c|c|}
\hline Study & eczema & $\begin{array}{l}\text { BCG } \\
\text { Total }\end{array}$ & eczema & $\begin{array}{c}\text { Control } \\
\text { Total }\end{array}$ & \multicolumn{3}{|c|}{ Odds Ratio } & OR & $95 \%-\mathrm{CI}$ & Weight (\%) \\
\hline \multicolumn{11}{|l|}{ developed country } \\
\hline Alm 1997 & 58 & 216 & 107 & 358 & & + & & 0.86 & {$[0.59 ; 1.25]$} & 9.0 \\
\hline Gruber 2002 & 2548 & 20383 & 1695 & 18425 & & $:+$ & & 1.41 & {$[1.32 ; 1.50]$} & 12.2 \\
\hline Marks 2003 & 69 & 307 & 112 & 441 & & + & & 0.85 & {$[0.60 ; 1.20]$} & 9.4 \\
\hline Garcia 2005 & 527 & 6762 & 246 & 2828 & & +1 & & 0.89 & {$[0.76 ; 1.04]$} & 11.6 \\
\hline Miyake 2008 & 391 & 5567 & 15 & 150 & & $\rightarrow:$ & & 0.68 & {$[0.39 ; 1.17]$} & 6.9 \\
\hline Thestesen 2018 & 466 & 2052 & 495 & 1952 & & + & & 0.86 & {$[0.75 ; 1.00]$} & 11.7 \\
\hline Mohrenschiager 2007 & 158 & 1221 & 41 & 248 & & + & & 0.75 & {$[0.52 ; 1.09]$} & 9.0 \\
\hline Gruber 2001 & 27 & 92 & 241 & 682 & & $\rightarrow$ & & 0.76 & {$[0.47 ; 1.22]$} & 7.7 \\
\hline Steenhuis 2008 & 39 & 62 & 31 & 59 & & + & & 1.53 & {$[0.74 ; 3.16]$} & 5.1 \\
\hline Random effects model & & 36662 & & 25143 & & & & 0.92 & {$[0.73 ; 1.17]$} & 82.5 \\
\hline \multicolumn{11}{|c|}{ Heterogeneity: $I^{2}=90 \%, \tau^{2}=0.0973, p<0.01$} \\
\hline \multicolumn{11}{|l|}{ developing country } \\
\hline Cunha 2004 & 100 & 637 & 56 & 276 & & + & & 0.73 & {$[0.51 ; 1.05]$} & 9.1 \\
\hline Soysal 2008 & 24 & 290 & 6 & 71 & & 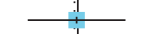 & & 0.98 & {$[0.38 ; 2.49]$} & 3.7 \\
\hline Peleteiro 2018 & 7 & 46 & 1 & 29 & & 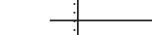 & & -5.03 & {$[0.58 ; 43.18]$} & 0.9 \\
\hline Singh 2013 & 39 & 7792 & 5 & 1700 & & + & & 1.71 & {$[0.67 ; 4.33]$} & 3.7 \\
\hline Random effects model & & 8765 & & 2076 & & $<$ & & 1.09 & {$[0.60 ; 1.96]$} & 17.5 \\
\hline \multicolumn{11}{|c|}{ Heterogeneity: $I^{2}=46 \%, \tau^{2}=0.1614, p=0.13$} \\
\hline Random effects model & & 45427 & & 27219 & & $\Leftrightarrow$ & & 0.94 & {$[0.76 ; 1.16]$} & 100.0 \\
\hline \multicolumn{5}{|c|}{$\begin{array}{l}\text { Heterogeneity: } I^{2}=86 \%, \tau^{2}=0.0972, p<0.01 \\
\text { Test for subgroup differences: } \chi_{1}^{2}=0.26, \mathrm{df}=1(p=0.61)\end{array}$} & 0.1 & 0.512 & 10 & & & \\
\hline
\end{tabular}

FIGURE 5: Forest plot of the association between BCG vaccination and eczema. Random effects model forest plot shows ORs and 95\% CIs for the association between BCG vaccination and eczema. $\mathrm{OR}=1.09,95 \%$ CI 0.60 to 1.96 . M-H $=$ Mantel-Haenszel; OR=odds ratio; $\mathrm{CI}=$ confidence interval.

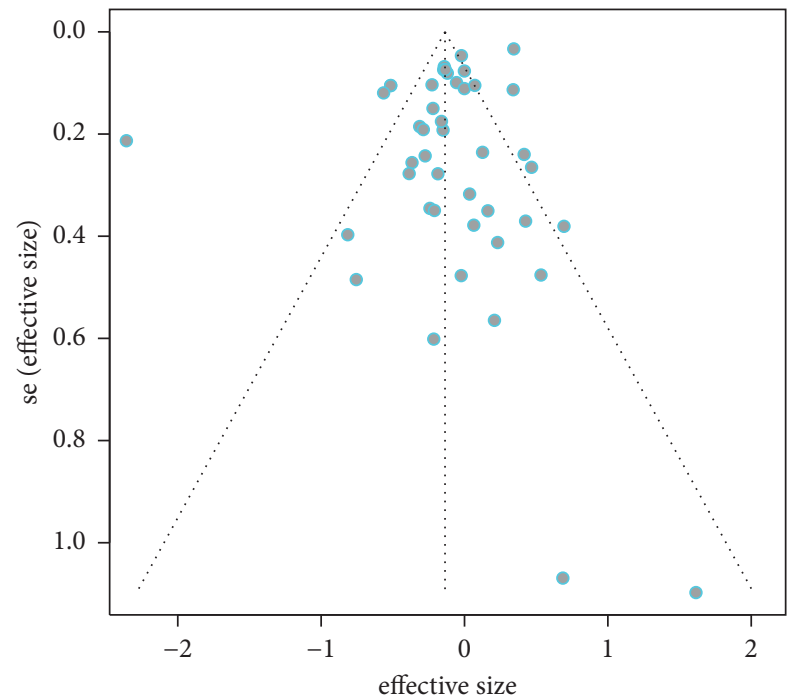

FIGURE 6: Funnel plot for the association between BCG vaccination and total atopic diseases. See Table 1 for reference citations. $\mathrm{SE}=$ standard error. $p=0.074$.

severity of atopic diseases, especially for eczema in the included studies, the applicability of the findings of children with varying degrees of severity is therefore compromised. This may have influenced the resulting protective effects of BCG vaccination in eczema and rhinitis.

Our meta-analysis has several strengths. Compared with a similar meta-analysis [19], six new articles were included in this paper. Each study sample size in this meta-analysis was assessed by two independent authors, so the result can be more accurate than others with low quality [50]. Furthermore, we followed the recommendations of the Cochrane Collaboration and PRISMA statement, including a priori protocol. Comprehensive assessment of the study quality was achieved by using STROBE checklist for the cohort studies and JADAD scale for the RCT study.

As with all systematic reviews, we may have failed to identify some studies, especially those with negative results, several studies are missing in this meta-analysis, including RCTs concerning neonatal BCG vaccination and wheeze and other possibly atopic diseases. Therefore, this may have influenced our findings. Finally, the length of time that the early effective protection from BCG vaccination lasts remains unanswered. The age of participants in this review may partially explain the result of the protective effect of BCG vaccination. In Linehan's study, it was shown that any benefits of BCG vaccine are likely to be transient [51]. Therefore, a large proportion of the protection from BCG vaccination may not be attributed to a reduction in the risk of atopy. Nonetheless, it can be confirmed that BCG vaccination in early childhood does reduce the risk of developing asthma in early life.

\section{Conclusion}

Our results provide evidence that BCG protects against the risk of atopic diseases with the most protective effects on asthma occurrence from multiple epidemiologically different settings. Our results also suggest that that BCG 
vaccination in early childhood is associated with reduced risk of atopic disease, especially in developed countries.

\section{Abbreviations}

$\begin{array}{ll}\text { BCG: } & \text { Bacille Calmette-Guerin } \\ \text { CI: } & \text { Confidence intervals } \\ \text { OR: } & \text { Odds ratios } \\ \text { PRISMA: } & \begin{array}{l}\text { Preferred Reporting Items for Systematic } \\ \text { Reviews and Meta-Analyses }\end{array} \\ \text { CENTRAL: } & \text { Cochrane Central Register of Controlled Trials } \\ \text { MOOSE: } & \begin{array}{l}\text { Meta-Analysis of Observational Studies in } \\ \text { Epidemiology }\end{array} \\ \text { IL: } & \text { Interleukin } \\ \text { TH: } & \text { T-helper cell. }\end{array}$

\section{Data Availability}

All data generated or analyzed during this study are included in this published article and its supplementary information files.

\section{Conflicts of Interest}

The authors have no conflicts of interest.

\section{Authors' Contributions}

K. Z., S. X., C. C., and P. F. conceived and designed this study. K. Z. and P.M. performed literature research, assessed study details, and evaluated study quality. Q. Z. and W. L. performed the statistical analysis. K. Z. and X. J. drafted the manuscript with the support of R. H., C. C., and P. F. All the authors gave final approval of this manuscript.

\section{Acknowledgments}

This research was supported by the Public Welfare Projects of Ningbo, China (no. 202002N3190), The Major Science and Technology Program for Medicine and Health in Zhejiang Province (no. WKJ-ZJ-2012), the Program for the National Natural Science Foundation of China (no. 81700025), Zhejiang Provincial Natural Science Foundation of China under Grant no. LY18H110003, the Foundation of Science and Technology Department of Zhejiang Province (no. 2018C37101), Zhejiang Province Public Welfare Technology Application Research Project of China (no. LGD19H110001), the grant of Social Development of Science and Technology Project of Ningbo (no. 2014C50086), and Ningbo Pan 3315 Project of Innovative Individual (no. 2020B-31-G).

\section{Supplementary Materials}

The search strategy suitable for PubMed is provided in Appendix 1. The PRISMA checklist includes the Systematic Reviews and Meta-Analyses (PRISMA) guidelines. (Supplementary Materials)

\section{References}

[1] L. A. Reynolds and B. B. Finlay, "Early life factors that affect allergy development," Nature Reviews Immunology, vol. 17, no. 8, pp. 518-528, 2017.

[2] S. E. Anderson, L. Weatherly, and H. L. Shane, "Contribution of antimicrobials to the development of allergic disease," Current Opinion in Immunology, vol. 60, pp. 91-95, 2019.

[3] M. Akdis, "New treatments for allergen immunotherapy," The World Allergy Organization journal, vol. 7, no. 1, p. 23, 2014.

[4] A. Daschner and J. González Fernández, "Allergy in an evolutionary framework," Journal of Molecular Evolution, vol. 88, no. 1, pp. 66-76, 2020.

[5] M. Sánchez-Borges, B. L. Martin, A. M. Muraro et al., "The importance of allergic disease in public health: an icaall statement," World Allergy Organization Journal, vol. 11, p. 8, 2018.

[6] L. Henriksen, J. Simonsen, A. Haerskjold et al., "Incidence rates of atopic dermatitis, asthma, and allergic rhinoconjunctivitis in Danish and Swedish children," The Journal of Allergy and Clinical Immunology, vol. 136, no. 2, pp. 360-366, 2015, e362.

[7] I. Delany, R. Rappuoli, and E. De Gregorio, "Vaccines for the 21st century," EMBO Molecular Medicine, vol. 6, no. 6, pp. 708-720, 2014.

[8] M. Ilyas, S. Afzal, J. Ahmad, S Alghamdi, and M Khurram, "The resurgence of measles infection and its associated complications in early childhood at a tertiary care hospital in peshawar, Pakistan," Polish Journal of Microbiology, vol. 69, pp. 1-8, 2020.

[9] F. Amat, P. Saint-Pierre, E. Bourrat et al., "Early-onset atopic dermatitis in children: which are the phenotypes at risk of asthma? Results from the orca cohort," PloS One, vol. 10, no. 6, Article ID e0131369, 2015.

[10] M. R. Odent, E. E. Culpin, and T. Kimmel, "Pertussis vaccination and asthma: is there a link?" Journal of the American Medical Association: The Journal of the American Medical Association, vol. 272, no. 8, pp. 592-593, 1994.

[11] M. El-Zein, F. Conus, A. Benedetti, D. Menzies, M.-E. Parent, and M.-C. Rousseau, "Association between Bacillus calmetteguérin vaccination and childhood asthma in the quebec birth cohort on immunity and health," American Journal of Epidemiology, vol. 186, no. 3, pp. 344-355, 2017.

[12] T. M. McKeever, S. A. Lewis, C. Smith, and R. Hubbard, "Vaccination and allergic disease: a birth cohort study," American Journal of Public Health, vol. 94, no. 6, pp. 985-989, 2004.

[13] E. L. Hurwitz and H. Morgenstern, "Vaccination and risk of allergic disease," American Journal of Public Health, vol. 1, p. 6, 2005.

[14] L. Nilsson, N.-I. M. Kjellman, and B. Björkstén, "Allergic disease at the age of 7 Years after pertussis vaccination in infancy," Archives of Pediatrics and Adolescent Medicine, vol. 157, no. 12, pp. 1184-1189, 2003.

[15] C. A. G. Timmermann, C. E. Osuna, U. Steuerwald, P. Weihe, L. K. Poulsen, and P. Grandjean, "Asthma and allergy in children with and without prior measles, mumps, and rubella vaccination," Pediatric Allergy and Immunology, vol. 26, no. 8, pp. 742-749, 2015.

[16] T. H. M. Ottenhoff and S. H. E. Kaufmann, "Vaccines against tuberculosis: where are we and where do we need to go?" PLoS Pathogens, vol. 8, no. 5, Article ID e1002607, 2012.

[17] M.-C. Rousseau, M.-E. Parent, and Y. St-Pierre, "Potential health effects from non-specific stimulation of the immune 
function in early age: the example of bcg vaccination," $P e$ diatric Allergy and Iimmunology, vol. 19, no. 5, pp. 438-448, 2008.

[18] L. G. Stensballe, S. Sørup, P. Aaby et al., "Bcg vaccination at birth and early childhood hospitalisation: a randomised clinical multicentre trial," Archives of Disease in Childhood, vol. 102, no. 3, pp. 224-231, 2017.

[19] D. L. Arnoldussen, M. Linehan, and A. Sheikh, "Bcg vaccination and allergy: a systematic review and meta-analysis," The Journal of Allergy and Clinical Immunology, vol. 127, no. 1, pp. 246-253, 2011.

[20] D. F. Stroup, J. A. Berlin, S. C. Morton et al., "Meta-analysis of observational studies in EpidemiologyA proposal for reporting," Journal of the American Medical Association, vol. 283, no. 15, pp. 2008-2012, 2000.

[21] D. Moher, A. Liberati, J. Tetzlaff, D. G. Altman, and PRISMA Group, "Preferred reporting items for systematic reviews and meta-analyses: the prisma statement," Annals of Internal Medicine, vol. 151, no. 4, pp. 264-269, 2009, W264.

[22] J. P. T. Higgins and S. G. Thompson, "Quantifying heterogeneity in a meta-analysis," Statistics in Medicine, vol. 21, no. 11, pp. 1539-1558, 2002.

[23] S. S. Cunha, A. A. Cruz, I. Dourado, M. L. Barreto, L. D. A. Ferreira, and L. C. Rodrigues, "Lower prevalence of reported asthma in adolescents with symptoms of rhinitis that received neonatal bcg," Allergy, vol. 59, no. 8, pp. 857-862, 2004.

[24] L. García-Marcos, M. Morales Suárez-Varela, I. Miner Canflanca et al., "Bcg immunization at birth and atopic diseases in a homogeneous population of Spanish schoolchildren," International Archives of Allergy and Immunology, vol. 137, no. 4, pp. 303-309, 2005.

[25] C. GrÃ ber, G. Meinlschmidt, R. Bergmann, U. Wahn, and K. Stark, "Is early bcg vaccination associated with less atopic disease? An epidemiological study in German preschool children with different ethnic backgrounds," Pediatric Allergy and Immunology, vol. 3, pp. 177-181, 2002.

[26] M. F. Linehan, T. L. Frank, M. L. Hazell et al., "Is the prevalence of wheeze in children altered by neonatal bcg vaccination?" The Journal of Allergy and Clinical Immunology, vol. 119, no. 5, pp. 1079-1085, 2007.

[27] G. B. Marks, K. Ng, J. Zhou et al., "The effect of neonatal bcg vaccination on atopy and asthma at age 7 to 14 years: an historical cohort study in a community with a very low prevalence of tuberculosis infection and a high prevalence of atopic disease," The Journal of Allergy and Clinical Immunology, vol. 111, no. 3, pp. 541-549, 2003.

[28] Y. Miyake, M. Arakawa, K. Tanaka, S. Sasaki, and Y. Ohya, "Tuberculin reactivity and allergic disorders in schoolchildren, okinawa, Japan," Clinical and Experimental Allergy, vol. 38, no. 3, pp. 486-492, 2008.

[29] M. Möhrenschlager, V. M. Haberl, U. Krämer, H. Behrendt, and J. Ring, "Early bcg and pertussis vaccination and atopic diseases in 5- to 7-year-old preschool children from augsburg, Germany: results from the miriam study," Pediatric Allergy and Immunology, vol. 18, no. 1, pp. 5-9, 2007.

[30] M. Mommers, M. Weishoff-Houben, G. M. H. Swaen et al., "Infant immunization and the occurrence of atopic disease in Dutch and German children: a nested case-control study," Pediatric Pulmonology, vol. 38, no. 4, pp. 329-334, 2004.

[31] A. Pahari, S. Welch, and S. Lingam, "Bcg, tuberculin skin-test results and asthma prevalence in school children in north london," Indian Pediatrics, vol. 3, pp. 254-258, 2002.
[32] L. M. Thøstesen, J. Kjaergaard, G. T. Pihl et al., "Neonatal bcg vaccination and atopic dermatitis before 13 months of age: a randomized clinical trial," Allergy, vol. 73, no. 2, pp. 498-504, 2018.

[33] J. S. Alm, G. Lilja, G. Pershagen, and A. Scheynius, "Early bcg vaccination and development of atopy," Lancet, vol. 350, no. 9075, pp. 400-403, 1997.

[34] C. Grüber, M. Kulig, R. Bergmann, I. Guggenmoos-Holzmann, U. Wahn, and MAS-90 Study Group, "Delayed hypersensitivity to tuberculin, total immunoglobulin e, specific sensitization, and atopic manifestation in longitudinally followed early bacille calmette-guérin-vaccinated and nonvaccinated children," Pediatrics, vol. 107, no. 3, p. E36, 2001.

[35] A. Soysal, N. Bahçeciler, I. Barlan, and M. Bakir, "Lack of an inverse association between tuberculosis infection and atopy: by t-cell-based immune assay (rd1-elispot)," Pediatric Allergy and Immunology, vol. 19, no. 8, pp. 709-715, 2008.

[36] T. J. Steenhuis, W. M. van Aalderen, N. Bloksma et al., "Bacille-calmette-guerin vaccination and the development of allergic disease in children: a randomized, prospective, singleblind study," Clinical and Experimental Allergy: Journal of the British Society for Allergy and Clinical Immunology, vol. 38, no. 1, pp. 79-85, 2008.

[37] K. Wickens, J. Crane, T. Kemp et al., "A case-control study of risk factors for asthma in New Zealand children," Australian \& New Zealand Journal of Public Health, vol. 25, no. 1, pp. 44-49, 2001.

[38] S. S. Park, E. Y. Heo, D. K. Kim, H. S. Chung, and C.-H. Lee, "The association of bcg vaccination with atopy and asthma in adults," International Journal of Medical Sciences, vol. 12, no. 8, pp. 668-673, 2015.

[39] T. S. Peleteiro, E. S. Oliveira, E. L. Conceição et al., "Impact of Bacille Calmette-Guérin revaccination on serum IgE levels in a randomized controlled trial," Revista da Sociedade Brasileira de Medicina Tropical, vol. 51, no. 1, pp. 94-98, 2018.

[40] M. Singh, R. R. Das, L. Kumar, and R. Kumar, "Bacille calmette-guérin vaccination is associated with lower prevalence of allergic diseases in Indian children," American Journal of Rhinology \& Allergy, vol. 27, no. 4, pp. e107-e112, 2013.

[41] L. M. Thøstesen, L. G. Stensballe, G. T. Pihl et al., "Neonatal bcg vaccination has no effect on recurrent wheeze in the first year of life: a randomized clinical trial," The Journal of Allergy and Clinical Immunology, vol. 140, no. 6, pp. 1616-e3, 2017.

[42] R. M. Harbord, M. Egger, and J. A. C. Sterne, "A modified test for small-study effects in meta-analyses of controlled trials with binary endpoints," Statistics in Medicine, vol. 25, no. 20, pp. 3443-3457, 2006.

[43] K. J. Erb, J. W. Holloway, A. Sobeck, H. Moll, and G. Le Gros, "Infection of mice with Mycobacterium bovis-Bacillus calmette-guérin (BCG) suppresses allergen-induced airway eosinophilia," Journal of Experimental Medicine, vol. 187, no. 4, pp. 561-569, 1998.

[44] C. B. Scanga and G. Le Gros, "Development of an asthma vaccine," Drugs, vol. 59, no. 6, pp. 1217-1221, 2000.

[45] C. Zuany-Amorim, E. Sawicka, C. Manlius et al., "Suppression of airway eosinophilia by killed mycobacterium vaccae-induced allergen-specific regulatory t-cells," Nature Medicine, vol. 8, no. 6, pp. 625-629, 2002.

[46] M. Yazdanbakhsh, P. G. Kremsner, and R. van Ree, "Allergy, parasites, and the hygiene hypothesis," Science, vol. 296, no. 5567, pp. 490-494, 2002. 
[47] M. Kowalewicz-Kulbat and C. Locht, "Bcg and protection against inflammatory and auto-immune diseases," Expert Review of Vaccines, vol. 16, no. 7, pp. 1-10, 2017.

[48] R. M. D. Bernsen, J. C. van der Wouden, N. J. D. Nagelkerke, and J. C. de Jongste, "Early life circumstances and atopic disorders in childhood," Clinical and Experimental Allergy, vol. 36, no. 7, pp. 858-865, 2006.

[49] M. A. Ferreira, J. M. Vonk, J. M. Vonk et al., "Shared genetic origin of asthma, hay fever and eczema elucidates allergic disease biology," Nature Genetics, vol. 49, no. 12, pp. 1752-1757, 2017.

[50] L. Lin, "Bias caused by sampling error in meta-analysis with small sample sizes," PLoS One, vol. 13, no. 9, Article ID e0204056, 2018.

[51] M. F. Linehan, U. Nurmatov, T. L. Frank, R. M. Niven, D. N. Baxter, and A. Sheikh, "Does bcg vaccination protect against childhood asthma? Final results from the manchester community asthma study retrospective cohort study and updated systematic review and meta-analysis," The Journal of Allergy and Clinical Immunology, vol. 133, no. 3, pp. 688-695, 2014, e614. 